\title{
An Elementary Identity with Cotangent Hyperbolic Function
}

\section{Prof. Dr. Raja Rama Gandhi and Edigles Guedes}

${ }^{1}$ Resource person in Math for Oxford University Press, Professor in Math, BITS-Vizag.

${ }^{2}$ World order Number Theorist, Pernambuco, Brazil.

LEMMA 1. For $a \in \mathbb{R} \backslash\{0\}$, then

$$
\frac{1}{a}=\sum_{k=1}^{\infty} \frac{1}{(a+k)(a+k-1)}
$$

Proof. We knew that

$$
\begin{gathered}
\frac{a}{b}=\prod_{k=1}^{\infty} \frac{(b+k)(a+k-1)}{(a+k)(b+k-1)} \\
\ln a-\ln b=\sum_{k=1}^{\infty} \ln \left[\frac{(b+k)(a+k-1)}{(a+k)(b+k-1)}\right] \\
\frac{\partial}{\partial a}(\ln a-\ln b)=\sum_{k=1}^{\infty} \frac{\partial}{\partial a} \ln \left[\frac{(b+k)(a+k-1)}{(a+k)(b+k-1)}\right] \\
\frac{1}{a}=\sum_{k=1}^{\infty} \frac{1}{(a+k)(a+k-1)}
\end{gathered}
$$

THEOREM 1. For $x \in \mathbb{R}$, then

$$
\begin{aligned}
& \frac{1}{\pi x}\left[\sum_{k=1}^{\infty}\left(\frac{1}{x^{2}+k}-\frac{1}{x^{2}+k-1}+\frac{1}{x+k-1}-\frac{1}{x+k}\right)\right]= \\
= & \frac{\cot (\pi x)}{x}+\sum_{k=1}^{\infty}\left[\frac{\cot \left(\pi \sqrt{x^{2}+k}\right)}{\sqrt{x^{2}+k}}-\frac{\cot \left(\pi \sqrt{x^{2}+k-1}\right)}{\sqrt{x^{2}+k-1}}\right] .
\end{aligned}
$$

Proof. We knew that

$$
\pi \cot (\pi x)=\frac{1}{x}+\sum_{n=1}^{\infty} \frac{2 x}{x^{2}-n^{2}} .
$$

Substituting (1) in (3), we encounter

$$
\begin{gathered}
\pi \cot (\pi x)=\sum_{k=1}^{\infty} \frac{1}{(x+k)(x+k-1)}+\sum_{n=1}^{\infty} 2 x \sum_{k=1}^{\infty} \frac{1}{\left(x^{2}-n^{2}+k\right)\left(x^{2}-n^{2}+k-1\right)} \\
=\sum_{k=1}^{\infty} \frac{1}{(x+k)(x+k-1)}+\sum_{k=1}^{\infty} \sum_{n=1}^{\infty} \frac{2 x}{\left(x^{2}-n^{2}+k\right)\left(x^{2}-n^{2}+k-1\right)} \\
=\sum_{k=1}^{\infty} \frac{1}{(x+k)(x+k-1)}-\sum_{k=1}^{\infty} \frac{x}{\left(x^{2}+k\right)\left(x^{2}+k-1\right)}+\pi x \sum_{k=1}^{\infty} \frac{\cot \left(\pi \sqrt{x^{2}+k-1}\right)}{\sqrt{x^{2}+k-1}} \\
-\pi x \sum_{k=1}^{\infty} \frac{\cot \left(\pi \sqrt{x^{2}+k}\right)}{\sqrt{x^{2}+k}},
\end{gathered}
$$


ergo,

$$
\frac{1}{\pi x}\left[\sum_{k=1}^{\infty}\left(\frac{1}{x^{2}+k}-\frac{1}{x^{2}+k-1}+\frac{1}{x+k-1}-\frac{1}{x+k}\right)\right]=\frac{\cot (\pi x)}{x}+\sum_{k=1}^{\infty}\left[\frac{\cot \left(\pi \sqrt{x^{2}+k}\right)}{\sqrt{x^{2}+k}}-\frac{\cot \left(\pi \sqrt{x^{2}+k-1}\right)}{\sqrt{x^{2}+k-1}}\right] . \square
$$

Note: But the equation $\sum_{k=1}^{\infty}\left[\frac{\cot \left(\pi \sqrt{x^{2}+k}\right)}{\sqrt{x^{2}+k}}-\frac{\cot \left(\pi \sqrt{x^{2}+k-1}\right)}{\sqrt{x^{2}+k-1}}\right]$ of right-hand side not converge. The Sir and I can modify the theorem to:

$$
\frac{\cot (\pi x)}{x}=\frac{1}{\pi x}\left[\sum_{k=1}^{\infty}\left(\frac{1}{x^{2}+k}-\frac{1}{x^{2}+k-1}+\frac{1}{x+k-1}-\frac{1}{x+k}\right)\right]-\pi x\left(\theta_{\cot (\pi x)}\right) ?
$$

for $\theta$ in function of $\cot (\pi x)$ ? 